\title{
EDITORIAL
}

\section{Enhancement of the Journal recognition}

The Journal of the National Science Foundation of Sri Lanka promotes and assists the publication of research papers of both local and foreign researchers working on all aspects of science and technology. The journal accepts submissions for publication under four categories; namely, Research Articles, Research Communications, Reviews and Correspondence.

During the last five years (2011-2016), the majority of submissions for publication has been research articles in areas related to biological sciences. The submissions in areas related to physical sciences, however, have shown a sharp increase in recent years, while the number of research articles related to biological sciences have remained at a steady level. The total number of published research articles has not shown much change over the years for both physical sciences as well as biological sciences.

Research Journals are generally evaluated using the Impact Factor. However, this tool must be used discreetly. Thomson Reuters determines the Impact Factor by dividing the number of current year citations to the source items published in that journal during the previous two years. It will be of special interest to the scientists and technologists who are contributors to the Journal and its general readership to know that the Impact Factor of the Journal of the National Science Foundation of Sri Lanka, which was 0.134 in 2010 has now increased to 0.277 in 2015 . This increasing trend is very encouraging.

The Journal credits this increasing trend to the authors/researchers, reviewers, members of the Editorial Board and members of the Editorial Office of the National Science Foundation of Sri Lanka. The increase in the Impact Factor is due to many reasons. One is the quality of research articles submitted for publication. The work of researchers is appreciated very much for their valuable efforts. The Journal also recognises the contributions made by the expert reviewers who provide the necessary advice and guidance to the Editorial Board and the Editor-in-Chief to make correct decisions and give appropriate feedback to the authors enabling them to improve the quality of their papers. The group of young officers in the Editorial Office also deserves credit for their commitment to ensure timely publication of the Journal.

It is essential to maintain the increasing trend of the Journal's Impact Factor. The increasing recognition of the Journal reflects the state of science in the country. It is also an important factor for the National Science Foundation of Sri Lanka, the authors /researchers and the reviewers who all contribute to the improvement of the Journal.

The researchers/authors have the responsibility of conducting and reporting original research work. When reporting their work, it is necessary to include appropriate citations of previous researchers both in the text and in the list of references. The Journal depends heavily on the corresponding author to ensure that all the researchers involved in a study are listed as co-authors correctly and also to confirm the accuracy of the final version of the article to be published. It is emphasised that the authorship is limited to those who have contributed significantly to the concept, design, implementation, analysis of data or interpretation of results. All others who have contributed substantially have to be given credit appropriately under acknowledgement. The corresponding author is also required to respond to all requests, queries and recommendations made by the reviewers, Editor-inChief and the Editorial Board. Disclosing of all financial support received for the research is also the responsibility of authors.

The reviewers have to play a major role in assuring transparency and integrity of the process of publishing 
research findings while being responsible, ethical and professional in all their review activities. Prompt action by reviewers contributes much to the satisfaction of the authors/researchers. Further, confidentiality of the manuscripts submitted for review is an essential factor that needs to be ensured by reviewers.

Nalini Ratnasiri 